\title{
A new frontier: Navigating hospital pharmacy practice during the COVID-19 pandemic
}

\author{
Nora BAIRAGDAR ${ }^{1}$ (D); Abigail Dee ANTIGUA ${ }^{1}$ (D), Mohammed AL-SAID ${ }^{1}$ (D) , A Kacee BARNETT ${ }^{1}$ (D), \\ Kayihura MANIGABA 1 * (D) \\ 1 Department of Clinical Pharmacy, North Florida Regional Medical Center, Gainesville, FL, USA. \\ * Corresponding Author. E-mail: kayihura.manigaba@gmail.com (K.M.).
}

Received: 10 November 2020 / Revised: 06 August 2021/ Accepted: 08 August 2021

The novel coronavirus, severe acute respiratory syndrome coronavirus 2 (SARS-CoV-2), first manifested in Wuhan, China in December 2019 as multiple cases of pneumonia with unknown etiology. This was the herald of an infectious catastrophe that would eventually affect millions of people across the world, claim countless lives, and uproot the very foundations of modern-day healthcare practice. Hospital pharmacists, alongside with physicians, nurses, and numerous other disciplines, are an integral part of the healthcare team that responded to this pandemic. The purpose of this article is to highlight the teamwork, determination, and innovativeness demonstrated by clinical pharmacists at a 510-bed community hospital in response to the coronavirus disease of 2019 (COVID-19). Pharmacists rose to the occasion to ensure that patients continue to receive the best therapy possible during this pandemic, and they supported other disciplines to ensure a collaborative response. Despite the unprecedented challenges posed to hospital pharmacy practice in the setting of COVID-19, our pharmacy team's response has resoundingly proven the resiliency of the human spirit, and shows that nothing is insurmountable in the face of collaboration, creativity, and an overwhelming desire to care for our community.

KEYWORDS: COVID-19 pandemic; hospital pharmacy; hospital pharmacist; collaboration; challenges.

\section{INTRODUCTION}

The novel coronavirus, severe acute respiratory syndrome coronavirus 2 (SARS-CoV-2), first manifested in Wuhan, China in December 2019 as multiple cases of pneumonia with unidentified etiology [1]. Unbeknownst to the rest of the world, this was the herald of an infectious catastrophe that would infect nearly 27 million people and claim over 900,000 lives globally as of September 2020 [2].

On March 11, 2020, the World Health Organization (WHO) officially declared the coronavirus disease of 2019 (COVID-19) a pandemic [3]. Two days later the United States designated COVID-19 as a national emergency [3]. The very foundations of modern-day healthcare practice were subsequently uprooted amidst the implementation of sweeping policy changes in an urgent attempt at adaptation to this nebulous new threat.

Alongside the physicians and nurses that have been rightfully lauded as heroes, hospital pharmacists are another integral component of the healthcare team that responded to this pandemic. Their duties are myriad, including being active members of hospital multidisciplinary teams, leading hospital committees, participating in patient rounds, acting as a repository of drug information and providing recommendations to other healthcare professionals, order verification to ensure the administration of safe and effective medication regimens to patients, and completing clinical consults that range from dosing antimicrobial agents to managing electrolytes and total parenteral nutrition [4]. Hospital pharmacists, as with all healthcare workers, experienced an abrupt alteration of their daily workflow with the emergence of COVID-19 and the rapid influx of operational, communication, and clinical changes that accompanied it [5].

What follows is an account of the challenges and triumphs experienced by the pharmacy team in a 510bed community hospital in Florida, United States during the early phase of COVID-19 pandemic, highlighting the teamwork, determination, and innovativeness required to successfully navigate hospital pharmacy practice during a crisis.

How to cite this article: Bairagdar N, Antigua AD, Al-Said M, Barnett AK, Manigaba K. A new frontier: navigating hospital pharmacy practice during the COVID-19 pandemic. J Res Pharm. 2021; 25(6): 823-827. 


\section{NAVIGATING COVID-19 PANDEMIC AS A HOSPITAL PHARMACIST}

\subsection{Pharmacy operations}

A strong operations team was the driving force that ensured our hospital continued to deliver the best care possible to patients during COVID-19 pandemic. Before the first case of COVID-19 was reported at the state level, an interdisciplinary team worked tirelessly every day to ensure that patient care standards would not be compromised in the event of a local outbreak. Law enforcement, emergency medical services (EMS), pharmacists, respiratory therapists, physicians, nurses, and representatives from nearby hospitals conducted tabletop exercises to discuss collaborative efforts needed to be successful once the pandemic inevitably reached our community [5].

The hospital pharmacy department was responsible for operational initiatives related to medications that could potentially lead to interruption in patient care, and also to ensure that all patients continue to receive the best therapeutic option despite all challenges that could arise. One method was a pharmacy team assessing potential manufacturing issues of medications that would be impacted by the pandemic, helping anticipate shortages before they occurred, and devising contingency plans. Upon experiencing a shortage of certain medications, the pharmacy department was able to expediently coordinate with other disciplines the implementation of alternative medications. For example, cisatracurium is the preferred neuromuscular blockage of choice for patients needing continuous infusion of a paralytic agent. Once our facility was not able to secure an adequate supply of cisatracurium, the pharmacy team immediately collaborated with physicians and other healthcare disciplines to switch to vecuronium as the alternative neuromuscular blockage agent for infusion [6].

The pharmacy operations team made several adjustments throughout the course of the COVID-19 pandemic as deemed necessary. Weekly operations meetings with other regional hospitals in our system were changed to daily so as to improve communication and encourage sharing of best practices in real-time. All inperson meetings were converted to an online platform to minimize the potential of COVID-19 transmission between employees and the consequent weakening of our workforce. The pharmacy department also implemented strategies to conserve personal protective equipment (PPE), such as outsourcing the compounding of select medications to an outside facility.

Multiple other operational changes were implemented to target employee safety. Before COVID-19, our hospital primarily utilized nebulizers for breathing treatments. To minimize the risk of infection through aerosol generation with nebulizer usage, the protocol was revised to instead prioritize inhaler use for patients with suspected or lab-confirmed COVID-19 and requiring breathing treatment. Prior to the pandemic, all satellite pharmacy personnel were required to respond to emergency codes on their floors - this responsibility was eventually centralized to one pharmacy team to limit possible COVID-19 exposure of multiple pharmacists. Pharmacy also instilled stringent measures to clean used crash carts and returned medications that could have been potentially exposed to a patient with COVID-19 to avoid cross-contamination and reduce viral spread.

The staffing model for the pharmacy department constantly evolved throughout this pandemic based on hospital needs and patient volume. During the initial local onset of COVID-19, decreases in the hospital census necessitated a temporary reassignment of roles. Pharmacists assisted nursing staff with responsibilities within their scope, and were also enlisted to participate in screening patients and visitors as per the hospital's infection control measures.

\subsection{Communications}

Transparent and routine communication is a crucial element in maintaining team morale, fostering trust, and reducing panic in overwhelming situations. With new COVID-19 information being circulated each day through the news, journal articles, and even word-of-mouth, hospital pharmacists may struggle with an excess of information. Therefore, it is important to establish reliable chains of communication in the hospital through which pharmacists can feel assured they are receiving accurate and relevant COVID-19 updates.

Communication from leadership is key to ensuring healthcare workers feel supported. Regular COVID19 updates are electronically communicated by facility administrators; these missives include the hospital census and current number of COVID-19 patients, PPE availability and conservation tips, masking and social distancing advice, and the process for COVID-19 testing. Furthermore, weekly online meetings are hosted by the company division's clinical pharmacy leaders. These sessions feature COVID-19 treatment guidance updates, review prominent new literature related to COVID-19 management, and provide an open platform for dialogue between corporate pharmacy leadership and local hospital pharmacy teams. 
Additionally, smaller-scale communications are needed to give hospital pharmacists the opportunity to initiate discussion and voice concerns to their colleagues and supervisors. The pharmacy department schedules two 15-minute virtual huddles each day in the morning and afternoon, which are attended by pharmacists, pharmacy technicians, residents, and students. These huddles serve to disseminate important policy and procedure changes or drug shortage information, to confer about important operational, patient care, and medication safety matters, and to perform a hand-off of any pressing patient care issues.

\subsection{Clinical practice}

One of the most arduous aspects of the COVID-19 pandemic for hospital pharmacists has been the rapidly-changing treatment recommendations. The United States Food and Drug Administration (FDA), for example, withdrew their March 2020 emergency use authorization (EUA) for hydroxychloroquine and chloroquine secondary to clinical trials finding both a lack of efficacy and risk for serious adverse events [7]. In May 2020, the WHO recommended against corticosteroid use in their interim guidance document for COVID-19, yet the randomized, controlled RECOVERY trial published in July 2020 supported dexamethasone usage in patients hospitalized with COVID-19 secondary to observed mortality benefit $[8,9]$.

Hospital pharmacists are fundamental in ensuring the use of the most up-to-date therapy for management of COVID-19 patients. The hospital antimicrobial stewardship program (ASP) committee consisting of infectious diseases (ID) pharmacists, physicians, and other key stakeholders creates and maintains a comprehensive COVID-19 treatment algorithm, incorporating recommendations from various regulatory and healthcare entities. The hospital monthly ASP meetings are led by the ID pharmacist and ID physician champion, which allows for multidisciplinary collaboration to revise the COVID-19 treatment recommendations as needed. Additionally, pharmacists have the opportunity to make significant clinical interventions at the order verification stage, such as intervening on polypharmacy issues with COVID-19 regimens. Pharmacists can discuss evidence-based treatment recommendations with physicians, discouraging off-label usage of medications that have not shown compelling evidence for treatment of COVID-19 [8]. As the use of vitamins and minerals such as ascorbic acid, vitamin D, and zinc becomes more prevalent (despite these supplements having inadequate data to recommend against or for their use in COVID-19), pharmacists can intervene by discussing available evidence with the ordering provider and discouraging the use of these supplements, especially when there are patient safety concerns such as drug-drug interactions with other medications, or potential adverse events [10].

Due to the high acuity of COVID-19 patients, collaboration of a multidisciplinary team is one of the factors necessary for successful care of these patients. In pre-COVID-19 times, multidisciplinary patient rounds were performed daily at bedside. However, due to the risk of viral transmission, the challenge of rounds became evident since there are representatives from all disciplines, including rehabilitation services, physicians, nurses, pharmacists, dieticians, and more. During the course of this pandemic, some multidisciplinary rounds were moved to a virtual platform to minimize the potential to spread the virus. Bedside multidisciplinary rounds in critical care areas with COVID-19 patients continued to occur in-person, but were restricted to physicians, midlevel practitioners, nurses, respiratory therapists, and pharmacists only.

Furthermore, COVID-19 patients require significant respiratory care and critical care management. Sedation and analgesia are used to assure that the patient stays comfortable while on mechanical ventilation, and also to prevent agitation while receiving other therapies such as rehabilitation therapy, proning, and chest physiotherapy. COVID-19 patients can become hemodynamically unstable during the course of hospitalization, which requires support through the use of vasopressors (i.e., norepinephrine, vasopressin), inotropes (i.e., milrinone, dobutamine), and/or fluids [10]. Due to these increased requirements, hospital pharmacists are a critical component of the multidisciplinary team taking care of COVID-19 patients.

One of the findings for patients with COVID-19 is the increased risk of venous thromboembolism (VTE), as some COVID-19 patients present with or progress to a hyper-inflammatory, hypercoagulability state [10]. For example, one of the signs that a COVID-19 patient is hyper-inflammatory is having an elevated D-Dimer (2-3x higher than normal). This finding necessitated a proactive approach of using pharmacologic thromboprophylaxis anticoagulation in hospitalized patients with COVID-19 or empiric therapeutic-intensity anticoagulation in patients suspected to be at very high risk of developing VTE [11-12]. Pharmacists are instrumental in screening COVID-19 patients for the aforementioned hyper-inflammatory state through the ordering of inflammatory lab markers. In addition to ensuring that appropriate anticoagulation is selected for patients with COVID-19, hospital pharmacists monitor the use of these anticoagulant medications, to ensure that patients are maintained at therapeutic goal and prevent adverse events such as bleeding [11]. 


\subsection{Challenges}

A significant number of challenges have been posed to hospital pharmacists by the COVID-19 pandemic. For the past few months, hospital policies and protocols have undergone frequent changes in a reflection of the fluctuating COVID-19 management recommendations by organizations such as the Centers for Disease Control and Prevention, FDA, and the National Institutes of Health. Providing daily communication from pharmacy leadership, implementing electronic health record (EHR) order sets for COVID-19 management, and using automated EHR alerts to monitor different aspects of COVID-19 therapy can help pharmacists feel supported and optimize the care provided to patients.

Another barrier to overcome during this pandemic has been the provision of experiential learning to pharmacy students and residents, attempting to simultaneously ensure their safety on rotations while not diminishing the quality of their education. Social distancing requirements have necessitated the removal of students from smaller satellite pharmacy locations in the hospital, compelling some learners and preceptors to conduct rotations remotely with reduced face-to-face time. Learners' active participation during bedside patient rounds were impacted, with rounds either transitioning to virtual modalities or limiting physical participants to only essential personnel. The move from in-person to online meetings also affected learner presentations and journal clubs, with audience engagement and discussion becoming more difficult to foster.

Recruitment has also faced difficulties. Decreased revenue has resulted at some point in hiring freezes in many healthcare systems, and low patient census numbers have caused many hospitals and systems to decrease their workforce during the early phase of this pandemic. Advertising for student and resident positions and job vacancies has been limited to only virtual forums. The interview process similarly underwent changes; the prevalence of phone and video interviews has increased, and onsite interviews may be hindered by measures to reduce risk of COVID-19 transmission, such as shorter interview durations, limited group interview capabilities due to social distancing, and difficulties gauging expressions and reactions due to mandated masks.

\section{CONCLUSION}

As with all pioneers traversing new and uncertain territory, hospital pharmacists have encountered unique challenges while responding to the COVID-19 pandemic. As described, pharmacists rose to the occasion to ensure that patients continue to receive the best therapy possible during this pandemic, and supported other disciplines to ensure a collaborative response. Many aspects of healthcare evolved rapidly during the COVID-19 pandemic, including our methods of communication, the flow of our day-to-day operations, and our clinical practice. Our experience during this pandemic has resoundingly served to demonstrate that nothing is insurmountable in the face of teamwork, creativity, and an overwhelming desire to care for one another and our community.

Acknowledgements: "This research was supported (in whole or in part) by HCA Healthcare and/or an HCA Healthcare affiliated entity. The views expressed in this publication represent those of the author(s) and do not necessarily represent the official views of HCA Healthcare or any of its affiliated entities."

Author contributions: Concept - N.B, A.A., M.A., A.K.B., K.M.; Design - N.B, A.A., K.M.; Supervision - N.B., K.M.; Resources - N.B, A.A., M.A., A.K.B., K.M..; Analysis and/or interpretation: N.B, A.A., M.A., A.K.B., K.M.; Literature Search - A.A., K.M..; Writing - N.B., A.A., K.M.; Critical Reviews - N.B, A.A., M.A., A.K.B., K.M.

Conflict of interest statement: "The authors declared no conflict of interest" in the manuscript.

\section{REFERENCES}

[1] Martellucci CA, Flacco ME, Cappadona R, et al. SARS-CoV-2 pandemic: An overview. Adv Biol Regul. 2020; 77: 111. [CrossRef]

[2] World Health Organization. Coronavirus disease (COVID-19) Weekly Epidemiological Update. WHO.int. Published September 6, 2020. Accessed September 7, 2020. https://www.who.int/docs/defaultsource/coronaviruse/situation-reports/20200907-weekly-epi-update-4.pdf?sfvrsn=f5f607ee_2.

[3] The American Journal of Managed Care. A Timeline of COVID-19 Developments in 2020. AJMC.com. Published July 3, 2020. Accessed September 5, 2020. https:/ /www.ajmc.com/view/a-timeline-of-covid19-developments-in-2020. 
[4] Hospital Pharmacy Europe. The role of the pharmacist in a multidisciplinary team. https://hospitalpharmacyeurope.com/news/editors-pick/ the-role-of-the-pharmacist-in-a-multidisciplinaryteam/

[5] Manigaba K, Gael MK. COVID-19: The Culprit, the People and Lessons Learned. HCA Healthcare Journal of Medicine. 2020; 1(4):247-250. [CrossRef]

[6] American Society of Hospital Pharmacists. Assessment of Evidence for COVID-19-Related Treatments. Updated September 17, 2020. Accessed September 20, 2020. https://www.ashp.org/-/media/assets/pharmacypractice/resource-centers/Coronavirus/docs/ASHP-COVID-19-Evidence-Table.ashx.

[7] U.S. Food and Drug Administration. COVID-19 Update: FDA Revokes Emergency Use Authorization for Chloroquine and Hydroxychloroquine. FDA.gov. Published June 15, 2020. Accessed September 7, 2020. https://www.fda.gov/news-events/press-announcements/coronavirus-covid-19-update-fda-revokes-emergencyuse-authorization-chloroquine-and.

[8] World Health Organization. Clinical management of COVID-19. WHO.int. Published May 27, 2020. Accessed September 7, 2020. https://www.who.int/publications/i/item/clinical-management-of-severe-acute-respiratoryinfection-when-novel-coronavirus-(ncov)-infection-is-suspected.

[9] Horby P, Lim WS, Emberson JR, et al. Dexamethasone in Hospitalized Patients with Covid-19 - Preliminary Report. N Engl J Med. 2020. [CrossRef]

[10] Pascarella G, Strumia A, Piliego C, et al. COVID-19 diagnosis and management: a comprehensive review. J Inter Med. 2020 Aug; 288(2):192-206. [CrossRef]

[11] Spyropoulos A, Weitz JI. Hospitalized COVID-19 patients and venous thromboembolism. Circulation. 2020; 142:12913. [CrossRef]

[12] Tang N, Bai H, Chen X, Gong J, Li D, Sun Z. Anticoagulant treatment is associated with decreased mortality in severe coronavirus disease 2019 patients with coagulopathy. J Thromb Haemost. 2020; 18(5): 1094-1099. [CrossRef]

This is an open access article which is publicly available on our journal's website under Institutional Repository at http://dspace.marmara.edu.tr. 